\title{
Correspondence
}

Letters should not exceed 400 words and should be typed double spaced (including the references) and be signed by all authors

TO THE EDITOR, Genitourinary Medicine

\section{Single dose ciprofloxacin to treat women with gonorrhoea}

Sir,

A single oral dose of ciprofloxacin is an effective treatment for men with urethral gonorrhoea. ${ }^{12}$ A small study of 10 women showed that a single oral dose of ciprofloxacin was satisfactory for treating women with gonorrhoea. ${ }^{3}$ In this unit during February 1987 to July 1988 ciprofloxacin 250 $\mathrm{mg}$ as a single oral dose, was given to 122 women with uncomplicated gonorrhoea. Neisseria gonorrhoeae was isolated by culture from the urethra or endocervix, or both, of all 122 women. In addition 29 of them had rectal gonorrhoea, six had pharyngeal gonorrhoea, and five had rectal and pharyngeal gonorrhoea. Tests of cure were carried out on two separate occasions (at the end of weeks 1 and 4) when urethral, endocervical, rectal, and pharyngeal swabs were again taken for culture. Fifty one patients received additional antibiotic treatment for concomitant genital infections during this period, and were excluded from the analysis.

Of the 71 women treated with ciprofloxacin alone, 49 attended on two or more occasions, 14 attended for one visit, and eight defaulted from follow up. Four of the 63 women who attended for follow up had a risk of reinfection, and three of them were culture positive. There were no treatment failures in the other 59 patients, who had 59 urogenital, 14 rectal, and five pharyngeal infections. All the isolates were sensitive to ciprofloxacin and penicillin, except one that required a minimum inhibitory concentration (MIC) of $2 \mathrm{mg} / \mathrm{l}$ penicillin, but did not produce $\beta$ lactamase.

Ciprofloxacin is a synthetic 4-quinolone derivative with a wide range of bactericidal activity. Our results show that $250 \mathrm{mg}$ given as a single dose is valuable in treating women with uncomplicated urogenital gonorrhoea. In addition this study shows that it offers the further advantages of oral administration, no apparent side effects, and the elimination of concomitant rectal and pharyngeal infections.

K Shanmugaratnam* MS Sprott
R S Pattman* A M Kearns $\dagger$ $P G$ Watson*

*Department of Genitourinary Medicine and + Regional Public Health Laboratory, Newcastle General Hospital,

Newcastle upon Tyne NE4 6BE

\section{References}

1 Loo PS, Ridgway GL, Oriel JD. Single dose ciprofloxacin for treating gonococcal infections in men. Genitourin Med 1985;61:302-5.

2 Scott GR, McMillan A, Young H. Ciprofloxacin versus ampicillin and probenecid in the treatment of uncomplicated gonorrhoea in men. J Antimicrob Chemother 1987;20: 117-21.

3 Avonts D, Fransen L, Vielfont J, Stevens A, Hendrickx K, Piot P. Treating uncomplicated gonococcal infection with $250 \mathrm{mg}$ or $100 \mathrm{mg}$ ciprofloxacin in a single oral dose. Genitourin Med 1988;64:134.

\section{TO THE EDITOR, Genitourinary Medicine}

Decreased in vitro susceptibility of penicillinase producing Neisseria gonorrhoeae to spectinomycin in Hong Kong

Sir,

Of 392 new patients attending this clinic from July to September 1987, 73 (18.6\%) had culture proved gonorrhoea that, in 33 , was caused by penicillinase producing Neisseria gonorrhoeae (PPNG) strains. All 33 patients were treated with single $2 \mathrm{~g}$ intramuscular doses of spectinomycin and followed up 48 hours and one week later. A patient was considered cured when all subsequent urethral or cervical cultures gave negative results. Treatment with spectinomycin cured 32 , and only one failed to respond. That was in line with the cure rate (not less than $95 \%$ (personal observation)) of spectinomycin in treating infections with PPNG and non-PPNG strains in this clinic for five years. The one patient in whom treatment failed was subsequently cured by $1 \mathrm{~g}$ cefotaxime intramuscularly. Though the reason for the treatment failure remains unknown, the PPNG strains obtained from this patient were less susceptible to spectinomycin than PPNG strains isolated in Hong Kong five to seven years ago, as did those obtained from other. patients in this study. In the present study, $19(58 \%)$ of the 33 strains required minimum inhibitory concentrations (MICs) of $32 \mathrm{mg} / \mathrm{l}$ spectinomycin, whereas only three $(12 \%)$ of 25 PPNG strains tested in 1981-3 in Hong Kong were inhibited by spectinomycin at that concentration ( $p<0.001$ in the $\chi^{2}$ test). Peeters et al also reported that gonococcal isolates in Africa showed diminished susceptibility to spectinomycin.' In their study the $\mathrm{MIC}_{50}$ and $\mathrm{MIC}_{90}$ of spectinomycin for 28 PPNG strains tested were both $32 \mathrm{mg} / \mathrm{l}$. Spectinomycin resistant $N$ gonorrhoeae clinical isolates are now well documented, ${ }^{2-4}$ and their emergence may have been accelerated in recent years by the widespread use of this drug to treat patients with primary gonorrhoea. ${ }^{4}$ Although spectinomycin resistant $N$ gonorrhoeae has not yet been isolated in Hong Kong, the susceptibility of clinical isolates to this drug should be monitored, and local clinicians should be aware what other effective alternative treatment regimens, such as newer cephalosporins, ${ }^{5}$ are available in case of spectinomycin treatment failure.

\section{HW Fung*}

WWS Ng†

*STD Clinic, 306A, Tung Ying Building, 100 Nathan Road, Kowloon, Hong Kong.

†Department of Extramural Studies, University of Hong Kong, Hong Kong.

\section{References}

1 Peeters M, Frost EH, Collet M, Ossari S, Yvert F, Ivanoff B. Changing antibiotic susceptibility of Neisseria gonorrhoeae in Franceville, Gabon. Antimicrob Agents Chemother 1987;31:1288-90.

2 Centers for Disease Control. Spectinomycinresistant Neisseria gonorrhoeae-world wide. MMWR 1982;31:637-8.

3 Centers for Disease Control. Spectinomycinresistant penicillinase-producing Neisseria gonorrhoeae. $M M W R$ 1983;32:51-2.

4 Boslego JW, Tramont EC, Takafuji ET, et al. Effect of spectinomycin use on the prevalence of spectinomycin-resistant and of penicillinase-producing Neisseria gonorrhoeae. $N$ Engl J Med 1987;317:212-8.

5 Mills J, Gottlieb A, Harrison WO. Treatment of gonorrhoea with first- and second-generation cephalosporins and other new $\beta$-lactam antibiotics. Sex Transm Dis 1986;13:203-6. 